\title{
Combined Spectroscopic/Computational Studies of Metal Centers in Proteins and Cofactors: Application to Coenzyme $B_{12}$
}

\author{
Thomas C. Brunold* \\ Werner Prize Winner 2003
}

\begin{abstract}
This article illustrates how the combined computational/spectroscopic methodology used in our studies of metal centers in proteins and cofactors can be applied to vitamin $B_{12}$ and its biologically relevant derivatives. The $\mathrm{B}_{12}$ cofactors have long fascinated chemists because of their complex structures and unusual reactivities in biological systems; however, their electronic absorption (Abs) spectra have remained largely unassigned. In this study, Abs, circular dichroism (CD), magnetic CD (MCD), and resonance Raman spectroscopic techniques are used to probe the electronic excited states of various $\mathrm{Co}^{3+} \mathrm{Cbl}$ species that differ with respect to their upper axial ligand. Spectroscopic data for each species are analyzed within the framework of time-dependent density functional theory (TD-DFT) to assign the major spectral features and to generate experimentally validated bonding descriptions. A simple model is presented that explains why the identity of the upper axial ligand has a major effect on the Co- $\mathrm{N}_{\text {ax }}$ bond strength, whereas the lower axial ligand does not appreciably modulate the nature of the $\mathrm{Co}-\mathrm{C}$ bond. Implications of these results with respect to enzymatic $\mathrm{Co}-\mathrm{C}$ bond activation are discussed.
\end{abstract}

Keywords: Bioinorganic chemistry $\cdot$ Coenzyme $B_{12} \cdot$ Density functional theory $\cdot$ Metalloenzymes $\cdot$ Spectroscopy

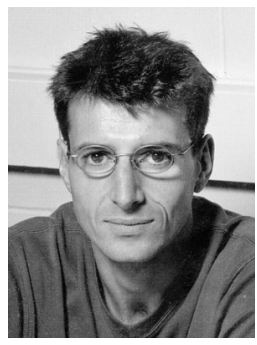

Thomas C. Brunold was born in Bern, Switzerland, in 1969. He received his $\mathrm{PhD}$ from the University of Bern in 1996, working with Hans U. Güdel on the design and spectroscopic characterization of new transitionmetal based near-IR laser materials. From 1997 to 1999, he was a postdoctoral fellow with Edward I. Solomon at Stanford University, studying binuclear iron and manganese centers in proteins and synthetic model complexes. He joined the faculty at the University of Wisconsin-Madison in 1999, where his research interests include spectroscopic and computational studies on superoxide dimsmutases and $\mathrm{Fe}-$, $\mathrm{Co}-$, and $\mathrm{Ni}$-containing enzymes and cofactors that form organometallic reaction intermediates.

${ }^{\star}$ Correspondence: Prof. T.C. Brunold

Department of Chemistry

1101 University Avenue

University of Wisconsin

Madison, WI 53706, USA

Tel.: +16082659056

Fax: +1 6082626143

E-Mail: brunold@chem.wisc.edu

http://www.chem.wisc.edu/main/people/faculty/

brunold.html

\section{Introduction}

The $\mathrm{B}_{12}$ cofactors methylcobalamin (MeCbl) and 5'-deoxyadenosylcobalamin (AdoCbl) have long fascinated chemists with their complex structures (Fig. 1) and unusual reactivities in biological systems [2-6]. Cobalamins possess a low-spin $\mathrm{Co}^{3+}$ center ligated equatorially by the four nitrogens of a highly substituted macrocycle (the corrin ring) and axially coordinated by a nitrogen from the tethered base 5,6-dimethylbenzimidazole (DMB) [7]. A wide range of ligands can occupy the upper axial position, such as $\mathrm{CN}, \mathrm{H}_{2} \mathrm{O}, \mathrm{Me}$, and Ado; however, only $\mathrm{MeCbl}$ and AdoCbl are enzymatically competent [8][9]. The MeCbldependent enzymes catalyze methyl-transfer reactions through heterolytic cleavage of the cofactor's $\mathrm{Co}-\mathrm{C}$ bond, yielding a protein-bound $\mathrm{Co}^{1+} \mathrm{Cbl}$ species [10-12]. Alternatively, enzymes utilizing AdoCbl catalyze radical-induced rearrangement reactions via homolytic cleavage of the $\mathrm{Co}-\mathrm{C}$ bond to produce $\mathrm{Co}^{2+} \mathrm{Cbl}$ and an organic radical centered on the 5' -carbon of the Ado moiety [9][13-17]. This Ado ${ }^{\bullet}$ radical then serves to abstract a hydrogen atom from substrate as the first step in a protein-mediated substrate rearrangement reaction. $\mathrm{B}_{12}$-dependent enzymes activate the organometallic $\mathrm{Co}-\mathrm{C}$ bond to a spectacular degree; a rate enhancement for bond homolysis by as much as 12 orders of magnitude has been reported for some enzymes [18-21].

Despite extensive studies of the $\mathrm{B}_{12}$ cofactors, fundamental questions regarding their electronic properties and reactivities remain largely unanswered. Electronic absorption (Abs) and circular dichroism (CD) spectroscopic techniques have been used extensively to probe changes in the geometric and electronic properties of the cofactor upon protein binding [22-24], but the lack of a suitable theoretical framework within which to interpret these changes greatly limited progress toward elucidation of enzymatic $\mathrm{Co}-\mathrm{C}$ bond activation mechanisms on the basis of spectroscopic data.

A 'typical' $\mathrm{Co}^{3+} \mathrm{Cbl} \mathrm{Abs} \mathrm{spectrum} \mathrm{(Fig.}$ 2 , top) is dominated by two features, the socalled $\alpha / \beta$ bands in the visible spectral region and the $\gamma$ band in the UV. Several additional, considerably weaker features termed $\mathrm{D}$ and $\mathrm{E}$ bands are sometimes observed between the $\alpha / \beta$ and $\gamma$ bands, the origin of which remained controversial. Such 'typical' Abs spectra are observed for nearly all $\mathrm{Co}^{3+} \mathrm{Cbl}$ species, such as vitamin $\mathrm{B}_{12}$ (cyanocobalamin, $\mathrm{CNCbl}$ ) and aquacobalamin $\left(\mathrm{H}_{2} \mathrm{OCbl}^{+}\right)$, in which the upper axial 


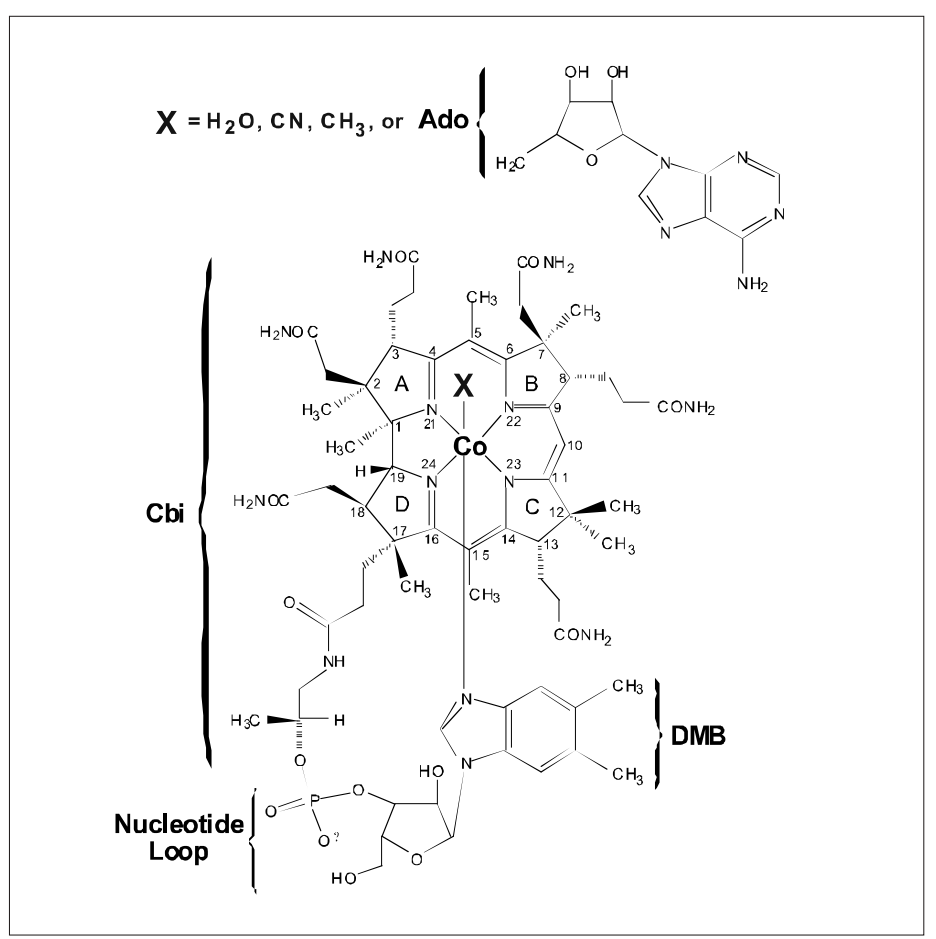

Fig. 1. Chemical structure and numbering scheme for $\mathrm{Co}^{3+} \mathrm{Cbls}$, where $\mathbf{X}$ indicates the upper axial ligand. Reprinted with permission from [1]. Copyright 2003 American Chemical Society.

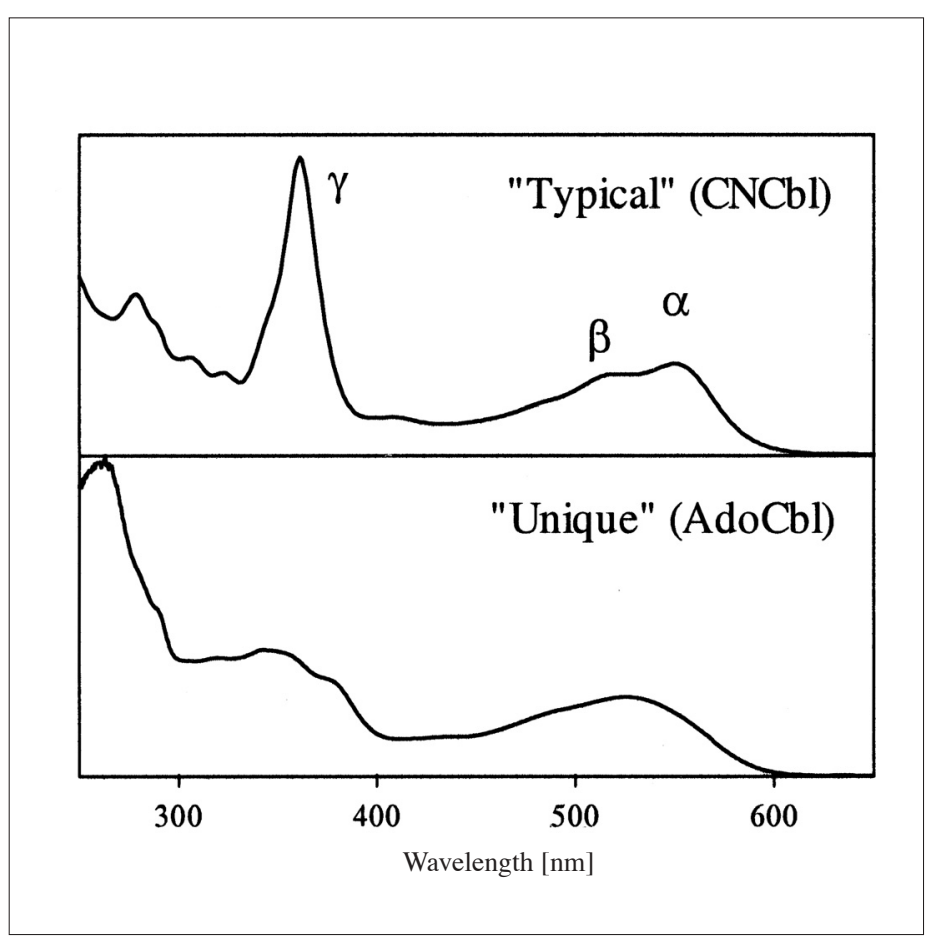

Fig. 2. Room temperature Abs spectra for aqueous solutions of CNCbl (top) and AdoCbl (bottom). Band designations are indicated in the 'typical' CNCbl Abs spectrum. Reprinted with permission from [1]. Copyright 2003 American Chemical Society. position is occupied by cyanide and water molecules, respectively (Fig. 1) [23][25]. In contrast, alkylcobalamins, such as $\mathrm{MeCbl}$ and AdoCbl, exhibit 'unique' Abs spectra that differ from 'typical' spectra in several ways (Fig. 2, top and bottom). Most strikingly, the intensity of the $\gamma$ band in the 'unique' spectra is redistributed over several similarly intense transitions in the UV spectral region. The fact that the $\mathrm{Co}^{3+} \mathrm{Cbl}$ Abs spectrum varies as a function of the 'upper' axial ligand even though it is dominated by corrin $\pi \rightarrow \pi^{*}$ transitions suggests that the axial ligands and the corrin macrocycle are electronically coupled; however, the mechanism by which this coupling occurs remained largely unexplored.

This article, which is based on a recent publication from our laboratory [1], illustrates how the combined spectroscopic/computational methodology used in our research can be applied to vitamin $\mathrm{B}_{12}$ and its biologically relevant derivatives. Abs, $\mathrm{CD}$, magnetic CD (MCD), and resonance Raman (RR) spectroscopic data are presented for a representative set of $\mathrm{Co}^{3+} \mathrm{Cbl}$ species whose upper axial ligands span the spectrochemical series, including $\mathrm{MeCbl}$, AdoCbl, CNCbl, and $\mathrm{H}_{2} \mathrm{OCbl}^{+}$. Spectroscopic data are analyzed within the framework of time-dependent density functional theory (TD-DFT) to assign key electronic transitions and to generate quantitative bonding descriptions. These studies permit detailed insight into the factors that distinguish $\mathrm{Co}^{3+} \mathrm{Cbls}$ exhibiting a 'typical' Abs spectrum from those displaying a 'unique' spectrum and afford a simple model that explains why the identity of the upper axial ligand has a major effect on the $\mathrm{Co}-\mathrm{N}_{\mathrm{ax}}$ bond strength, whereas the lower axial ligand does not appreciably modulate the nature of the $\mathrm{Co}-\mathrm{C}$ bond.

\section{Spectroscopic/Computational Methodology}

The combined spectroscopic/computational methodology employed in our research on metal centers in proteins and cofactors is based largely on the pioneering work of Solomon and coworkers [26][27]. The Table provides an overview of the methods used in our laboratory and the insights they provide into geometric and electronic structure. Several recent developments have been crucial to the success of our research; e.g. the vast improvements in computer technology that now permit DFT calculations to be performed on realistic active-site and cofactor models and the establishment of a theoretical framework by Neese and Solomon for the quantitative analysis of VTVH-MCD data for systems with spin $\mathrm{S} \geq 1 / 2$ (Table) [28].

\section{Results and Analysis}

\subsection{Spectroscopic Data}

\subsubsection{Abs, $C D$, and $M C D$}

Fig. 3 shows Abs, CD, and MCD spectra of three different $\mathrm{Co}^{3+} \mathrm{Cbl}$ species, in- cluding $\mathrm{MeCbl}, \mathrm{H}_{2} \mathrm{OCbl}^{+}$, and $\mathrm{CNCbl}$ (vi$\operatorname{tamin} \mathrm{B}_{12}$ ) [1]. Consistent with the diamagnetic ground state of $\mathrm{Co}^{3+} \mathrm{Cbls}$ (i.e. the $\mathrm{Co}^{3+}$ center has a low-spin $3 \mathrm{~d}^{6}$ electron configuration), all features in the MCD spectra of Fig. 3 were found to be temperature independent.

$\mathrm{H}_{2} \mathrm{OCbl}^{+}$: The Abs spectrum of $\mathrm{H}_{2} \mathrm{OCbl}^{+}$(Fig. 3B, top) shows the three sets of bands that characterize a 'typical' $\mathrm{Co}^{3+} \mathrm{Cbl}$ spectrum: the $\alpha / \beta$ bands previously assigned as the origin and a vibrational sideband of the corrin-based HOMO $\rightarrow$ LUMO (i.e. $\pi \rightarrow \pi^{*}$ ) transition polarized along the long axis of the corrin macrocycle $\left(C^{5} \ldots C^{15}\right.$ vector, Fig. 1) [25][29]; the $\mathrm{D} / \mathrm{E}$ bands that are relatively well resolved for this particular species; and the $\gamma$ band attributed to a corrin $\pi \rightarrow \pi^{*}$ transition polarized along the short corrin axis $\left(\mathrm{Co} \cdots \mathrm{C}^{10}\right.$ vector) [25][29]. The $\alpha / \beta$ region of the $C D$ spectrum (Fig. 3B, center) exhibits one band of large negative intensity that coincides with the $\alpha$ band in the Abs spectrum and several weak features of positive intensity, one of which occurs at lower energy than the $\alpha$ band. In the MCD spectrum (Fig. $3 \mathrm{~B}$, bottom) a single weak feature is observed in this region that coincides with the $\alpha$ band. The D/E bands coincide with the two negative features in the corresponding region of the MCD spectrum, whereas in the CD spectrum two relatively intense features that are offset relative to the $\mathrm{D} / \mathrm{E}$ bands (and, thus, must be associated with different electronic transitions) are observed in this region. Lastly, the $\gamma$ region of 
Table. Overview of spectroscopic and computational methods employed in our bioinorganic research ${ }^{\mathrm{a}}$

Method

Obtainable Parameters

Information Content

\section{Ground State}

Electron Paramagnetic Resonance (EPR)

$g$ values; axial (D) and rhombic $(E)$ zero-field splittings (ZFS)

Hyperfine couplings

Variable-temperature/varia- $\mathrm{g}$ values; ZFS parameters ble-field Magnetic Circular

Dichroism (VTVH MCD) Transition polarizations

Resonance Raman (RR) Vibrational frequencies

Isotope shifts

\section{Excited States}

Electronic Absorption (Abs) Transition energies and intensities (oscillator strengths)

Band shapes

Circular Dichroism (CD) Transition energies and intensities (rotational strengths)

Magnetic CD (MCD) Transition energies and intensities ( $A, B$, and $C$ terms)

$\mathrm{C} / \mathrm{D}$ ratio ( $\propto \mathrm{MCD} / \mathrm{Abs}$ intensity)

\section{Computations}

Density Functional Theory Energy-minimized metric (DFT)

parameters

Molecular orbital (MO) energies and compositions

Time-dependent (TD) DFT Predicted transition energies and Abs intensities

Semi-empirical INDO/S-Cl Predicted Spin Hamiltonian parameters ( $g$ values, $D$, and $\mathrm{E}$ ) and transition energies and Abs intensities
Structures and energies of hypothetical active-site models

Quantitative bonding description

Aids in evaluating hypothetical active-site models

Same as TD-DFT
Spin of ground state; ligand field (LF) splittings of metal $3 d$ orbitals and their relative covalencies

Spin distribution within molecule

See EPR above

Identification of ligand(s) involved in charge transfer (CT) transitions

Force constants

Band assignments; binding mode of exogenous ligands

LF splittings of metal $3 d$ orbitals; metal-ligand bond covalencies; orbitals

\section{Excited-state distortions}

ame as Abs; resolution of overabs spectrum, (a)

avs; resolution of overlapping Abs bands, nature of excited states

Metal character in transition

aSee [26] and [27] for excellent reviews of the spectroscopic methods typically employed in bioinorganic research.

the CD spectrum contains contributions from at least two transitions, as evidenced by the presence of two positive $\mathrm{CD}$ features in this region.

CNCbl: The Abs, CD, and MCD spectra of CNCbl (Fig. 3C) exhibit the same basic features as those of $\mathrm{H}_{2} \mathrm{OCbl}^{+}$; however, with several subtle differences. The $\alpha$ band is red-shifted by $760 \mathrm{~cm}^{-1}$ to $18080 \mathrm{~cm}^{-1}$, and there is no indication for the presence of a low-energy feature similar to the one observed in the $\mathrm{H}_{2} \mathrm{OCbl}^{+} \mathrm{CD}$ spectrum (Fig. 3B and 3C, center). The $\gamma$ region again reveals the presence of at least two electronic transitions, giving rise to the very intense Abs band with a well-developed shoulder on its high-energy side, which is red-shifted by $880 \mathrm{~cm}^{-1}$ with respect to the $\gamma$ band in the $\mathrm{H}_{2} \mathrm{OCbl}^{+}$spectra (Fig. 3B and 3C).

$\mathrm{MeCbl}$ : The dominant features in the 'unique' Abs spectrum of MeCbl (Fig. 3A, top) can be similarly classified as those observed in a 'typical' Cbl Abs spectrum; however, key differences exist that allow for experimental insight into the effects of the upper axial ligand on the corrinoid electronic structure. Importantly, the presence of the derivative-shaped features in the $\alpha / \beta$ region of the $\mathrm{CD}$ and $\mathrm{MCD}$ spectra (Fig. $3 \mathrm{~A}$, center and bottom) suggests that the prominent shoulder on the high-energy side of the $\beta$ band in the MeCbl Abs spectrum (Fig. 3A, top) corresponds to the origin of another electronic transition. Hence, the $\alpha / \beta$ region of the Abs spectrum differs from that of 'typical' $\mathrm{Co}^{3+} \mathrm{Cbl}$ spectra in that a second relatively intense electronic transition contributes to the Abs spectrum. However, the most unique aspect of the $\mathrm{MeCbl}$ Abs spectrum is that the intensity of the $\gamma$ band is redistributed over three partially resolved features in the UV region that carry similar intensities. Thus the number of electronic transitions in the $\gamma$ region, similar to the $\alpha / \beta$ region, also increases from the 'typical' to 'unique' $\mathrm{Co}^{3+} \mathrm{Cbl}$ spectra.

\subsubsection{RR Data}

Fig. 4 shows resonance Raman (RR) spectra of $\mathrm{H}_{2} \mathrm{OCbl}^{+}$and $\mathrm{MeCbl}$ obtained with $514.5 \mathrm{~nm}\left(19436 \mathrm{~cm}^{-1}\right)$ laser excitation. Despite the fact that the two species exhibit markedly different electronic Abs, CD, and MCD spectra (Fig. 3), their RR spectra are strikingly similar. In both cases the dominant features are due primarily to corrin-based vibrations, with the most intense band at $\sim 1500 \mathrm{~cm}^{-1}$ corresponding to the long-axis polarized corrin mode $v_{L A}$ depicted schematically in the inset of Fig. 4. This similarity indicates that substitution of the water ligand in $\mathrm{H}_{2} \mathrm{OCbl}^{+}$by a methyl group in $\mathrm{MeCbl}$ does not drastically alter intra-corrin bonding. Intriguingly, however, the 514.5-nm excited RR spectrum of $\mathrm{MeCbl}$ exhibits an additional band of significant intensity at $505 \mathrm{~cm}^{-1}$, previously attributed to the $\mathrm{Co}-\mathrm{C}$ stretching mode $v_{\text {Co-C }}[30][31]$. Enhancement of this mode upon excitation in resonance with a corrinbased $\pi \rightarrow \pi^{*}$ transition provides further evidence that the $\pi$ system of the corrin macrocycle and the $\mathrm{Co}-\mathrm{C}$ bond are electronically coupled.

\subsection{Spectral Analysis}

The Abs, CD, and MCD spectra presented in Fig. 3 were iteratively fit with the fewest possible number of Gaussian bands to resolve the major electronic transitions contributing to the Abs spectrum of each $\mathrm{Co}^{3+} \mathrm{Cbl}$ species investigated [1]. The results obtained from Gaussian deconvolutions of the spectra of $\mathrm{H}_{2} \mathrm{OCbl}^{+}$, a representative example of a $\mathrm{Co}^{3+} \mathrm{Cbl}$ species exhibiting a 'typical' Abs spectrum, and $\mathrm{MeCbl}$, representative of $\mathrm{Co}^{3+} \mathrm{Cbl}$ species displaying a 'unique' Abs spectrum, are shown in Fig. 5. While a detailed discussion of the results obtained from this analysis is beyond the scope of this article, the fits are shown here to demonstrate the complemen- 


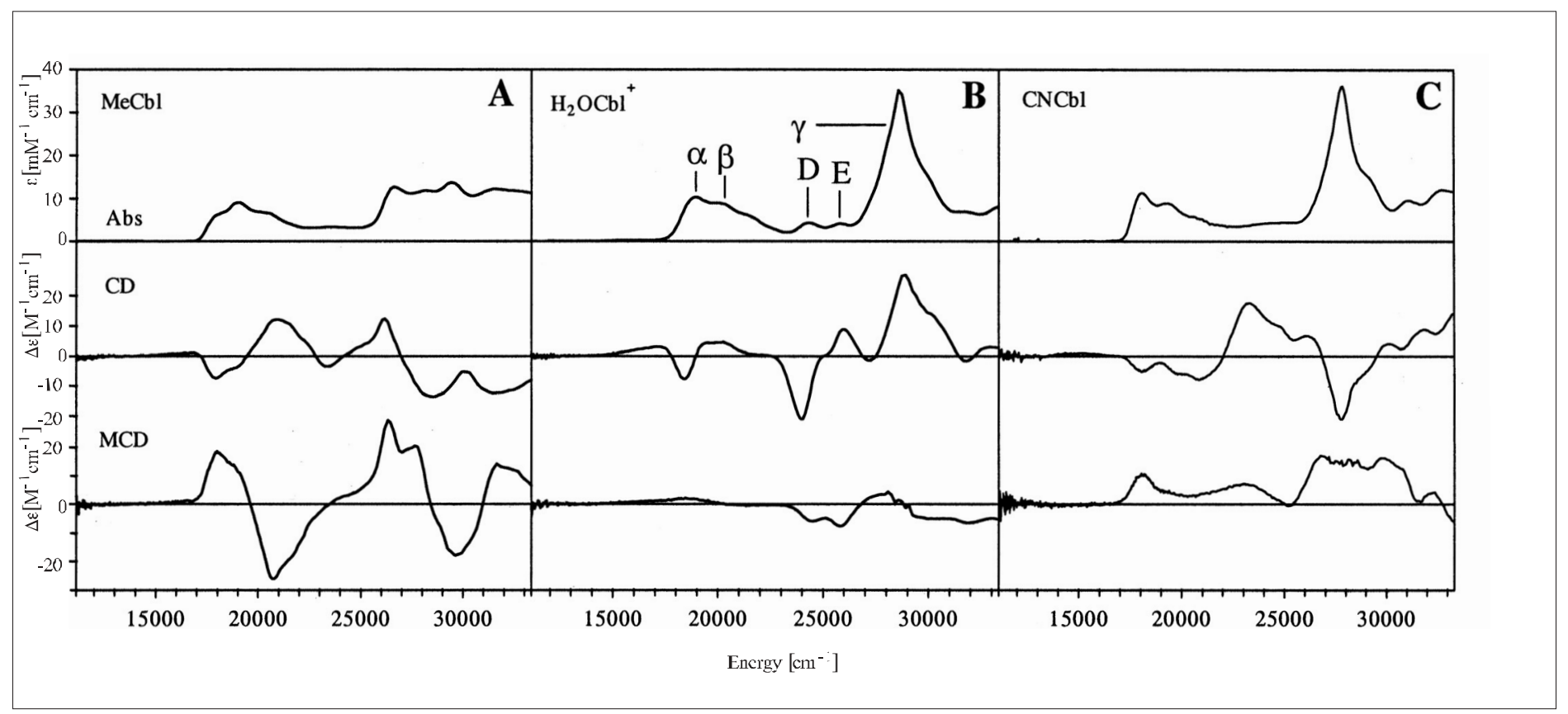

Fig. 3. Absorption (top), CD (center), and $7 \mathrm{~T} \mathrm{MCD} \mathrm{(bottom)} \mathrm{spectra} \mathrm{recorded} \mathrm{at} 4.5 \mathrm{~K}$ of MeCbl (A), $\mathrm{H}_{2} \mathrm{OCbl} \mathrm{I}^{+}(\mathrm{B})$, and CNCbl (C). Band designations are given for $\mathrm{H}_{2} \mathrm{OCbl}^{+}$. Reprinted with permission from [1]. Copyright 2003 American Chemical Society.

tary nature of $\mathrm{Abs}, \mathrm{CD}$, and $\mathrm{MCD}$ data. Clearly, it would be unreasonable to claim that 13 electronic transitions contribute to the spectral region between 15000 and $33000 \mathrm{~cm}^{-1}$ based solely on an analysis of the Abs spectrum. However, the fact that the intensities of electronic transitions are governed by different selection rules in Abs, $\mathrm{CD}$, and MCD spectra typically allows electronic transitions that are too weak to be

observed by one technique to be probed using one of the other two methods [26][27]. Knowledge of the exact number of electronic transitions contributing to the Abs spectrum of the species under investigation is crucial, as this information provides an excellent foundation for the rigorous evaluation of computations aimed at generating quantitative bonding descriptions (vide infra).

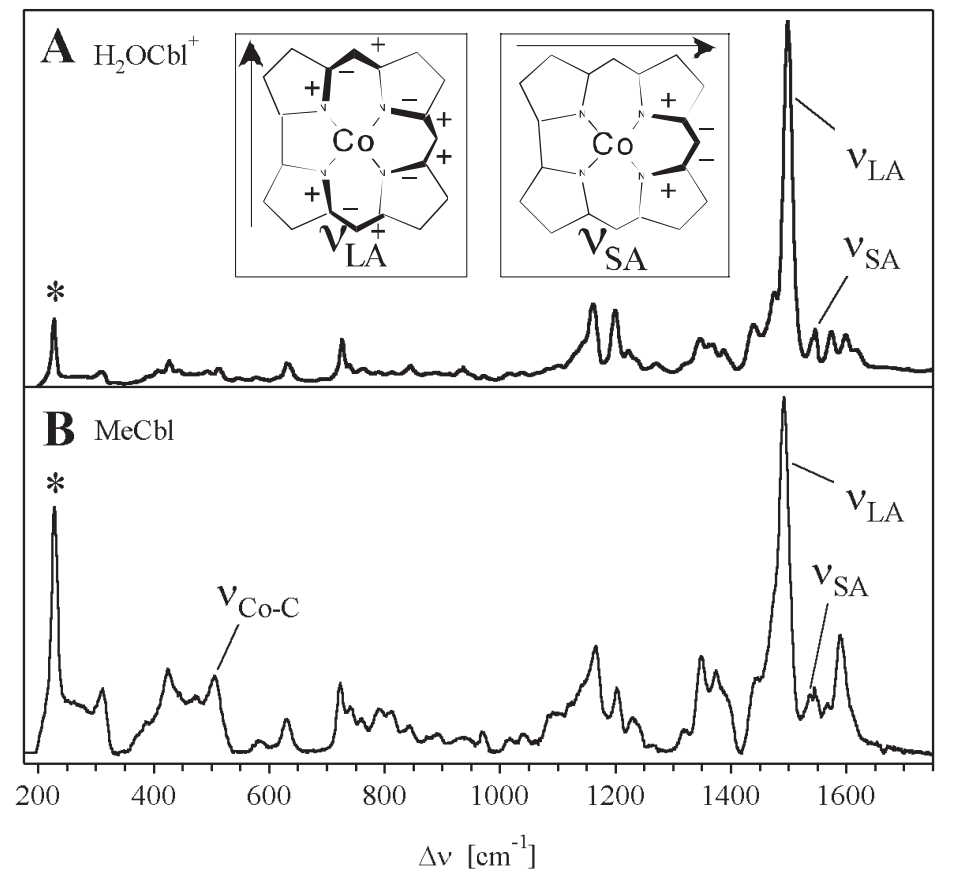

Fig. 4. $\mathrm{RR}$ spectra at $77 \mathrm{~K}$ of $\mathrm{H}_{2} \mathrm{OCbl}^{+}(\mathrm{A})$ and $\mathrm{MeCbl}(\mathrm{B})$ obtained with $514.5-\mathrm{nm}$ excitation. Insets: Normal mode descriptions and polarizations of $v_{\mathrm{LA}}$ (left) and $v_{\mathrm{SA}}$ (right). Reprinted with permission from [1]. Copyright 2003 American Chemical Society.

\subsection{Computational Data}

A number of computational studies of $\mathrm{Co}^{3+} \mathrm{Cbls}$ have been reported in the recent past, most of which employed the DFT method and relied on the success of geometry optimizations to validate the methodology used [32-34]. However, this approach has the inherent disadvantage that errors are necessarily introduced by using simplified $\mathrm{Co}^{3+} \mathrm{Cbl}$ models (note that despite significant recent advances in computer technology, treatment of the entire $\mathrm{Co}^{3+} \mathrm{Cbl}$ cofactor at the DFT level is still unfeasible). Instead, we generated our $\mathrm{Co}^{3+} \mathrm{Cbl}$ models based on high-resolution X-ray structures [35-37] and used our spectroscopic data in conjunction with the TD-DFT method [38-40] to validate the calculated bonding descriptions [1]. In this evaluation process, a series of TD-DFT calculations were performed on various cofactor models with a variety of functionals and basis sets to find the combination that yielded the best overall agreement between experimental and calculated Abs spectra of $\mathrm{H}_{2} \mathrm{OCbl}^{+}, \mathrm{CNCbl}$, and $\mathrm{MeCbl}$. While qualitatively similar results were obtained using pure DFT functionals, the B3LYP hybrid functional [41-43] proved most successful in this study as it compensates for the tendency of DFT to overestimate covalent contributions to metal-ligand bonding. To facilitate comparison with our spectroscopic data, TD-DFT computed transition energies and oscillator strengths were used to simulate Abs spectra assuming that each electronic transition gives rise to a Gaussian band of uniform width. Fig. 6 shows that the agreement between calculated and experimental Abs 


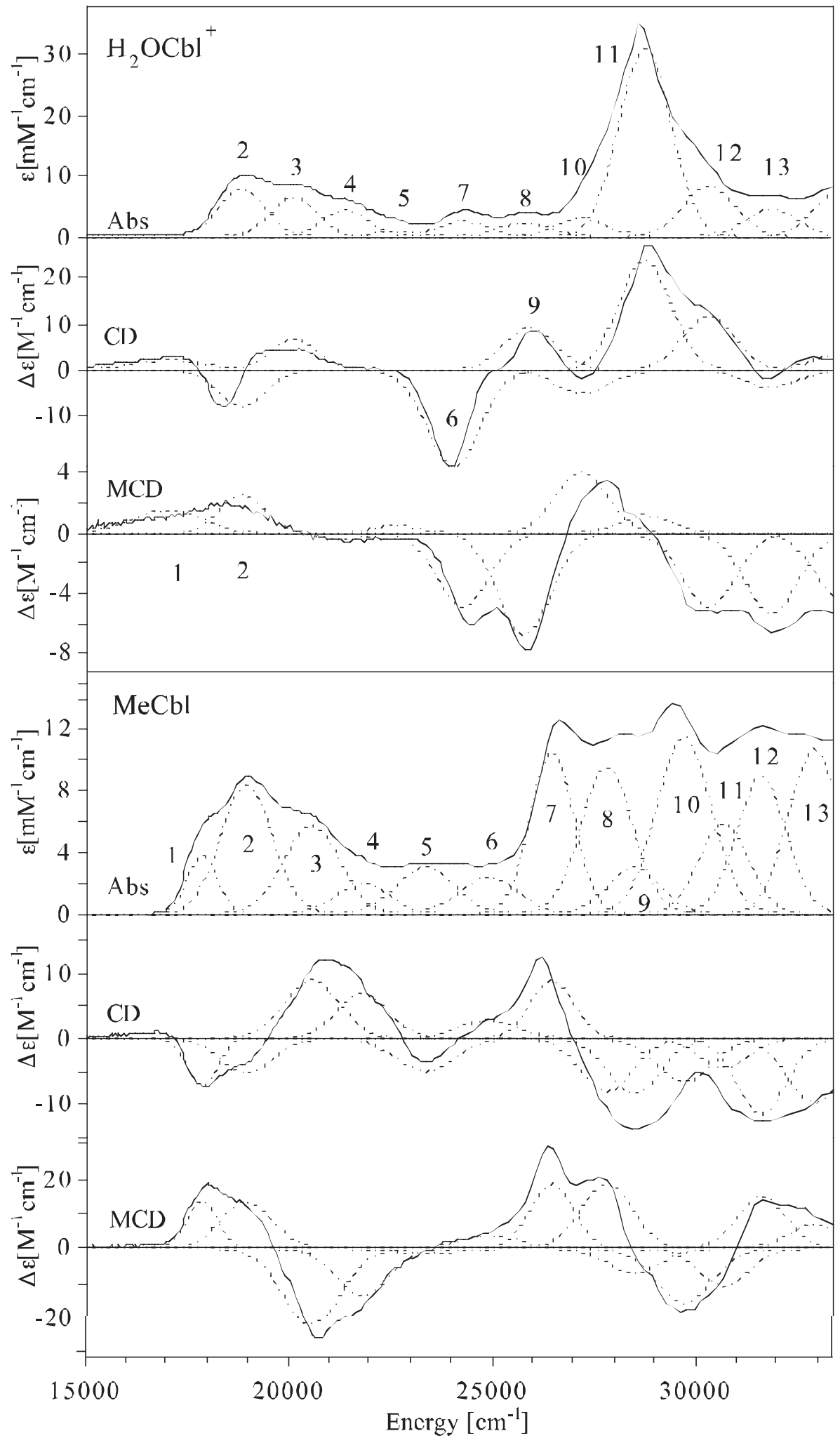

Fig. 5. Solid lines: $4.5 \mathrm{~K}$ Abs, $\mathrm{CD}$, and $7 \mathrm{~T} \mathrm{MCD}$ spectra of $\mathrm{H}_{2} \mathrm{OCbl}^{+}$(top 3 panels) and $\mathrm{MeCbl}$ (bottom 3 panels). Dotted lines: Gaussian deconvolutions of the experimental spectra. Note that bands 1,6, and 9 observed in the $\mathrm{CD}$ and MCD spectra of $\mathrm{H}_{2} \mathrm{OCbl}^{+}$do not appreciably contribute to the Abs spectrum. Reprinted with permission from [1]. Copyright 2003 American Chemical Society. spectra for all $\mathrm{Co}^{3+} \mathrm{Cbl}$ species investigated is quite remarkable.

$\mathrm{H}_{2} \mathrm{OCbl}^{+}$and $\mathrm{CNCbl}$ : For $\mathrm{H}_{2} \mathrm{OCbl}^{+}$and $\mathrm{CNCbl}$, our TD-DFT calculations predict one intense electronic transition in the visible spectral region that is polarized along the $C^{5} \cdots C^{15}$ vector of the corrin ring (Fig. 1) and one very intense transition in the UV that is polarized along $\mathrm{Co} \cdots \mathrm{C}^{10}$ vector. Thus the calculations almost quantitatively reproduce the main characteristics of the electronic transitions responsible for the $\alpha / \beta$ bands and the $\gamma$ band of 'typical' Abs spectra (Fig. 6A and 6B). Additional transitions carrying lower intensities are predicted near $25000 \mathrm{~cm}^{-1}$ where the D and E bands are observed in the experimental Abs spectra. Remarkably, the calculation on the $\mathrm{H}_{2} \mathrm{OCbl}^{+}$model also successfully reproduces the weak transition that gives rise to the positive feature on the low-energy side of the $\alpha$ band in the experimental CD spectrum (band 1 in Fig. 5, top).

$\mathrm{MeCbl}$ : Our TD-DFT computation on the MeCbl model (Fig. 6C) suggests the presence of two intense features in the $\alpha / \beta$ region of 'unique' $\mathrm{Co}^{3+} \mathrm{Cbl} \mathrm{Abs}$ spectra, consistent with the results from the Gaussian deconvolutions of our experimental spectra (bands $1 / 2$ and $3 / 4$ in Fig. 5, bottom). Significantly, our TD-DFT calculations also successfully reproduce the dramatic changes in the $\gamma$ region of the $\mathrm{Co}^{3+} \mathrm{Cbl}$ Abs spectrum upon substitution of the upper axial ligand by an alkyl group. The calculated 'unique' Abs spectrum of MeCbl shows multiple bands of similar intensities in the UV region where the single intense $\gamma$ band is predicted (and observed, see Fig. 3) in 'typical' $\mathrm{Co}^{3+} \mathrm{Cbl}$ spectra.

\subsection{Spectral Assignments}

As space limitations preclude a detailed discussion of band assignments that emerged from these studies [1], a brief summary of our general strategy that we typically follow to assign key spectral features is given instead. First, the full range of spectroscopic techniques available in our laboratory (Table) is used to experimentally probe the electronic structure of the metal center of interest (as outlined in section 3.1.). Next, a careful spectral analysis is carried out to resolve, and tentatively assign, the major electronic transitions (see section 3.2.). In the following step, computational models are generated and evaluated on the basis of the spectroscopic data and the findings from the corresponding spectral analysis (section 3.3.). Once satisfactory agreement between all calculated and experimental parameters is achieved, indicating that the computed electronic structure description is reasonable, meaningful band assignments can be made based on the computational results. In the present case, the fact that our TD-DFT computed Abs spec- 


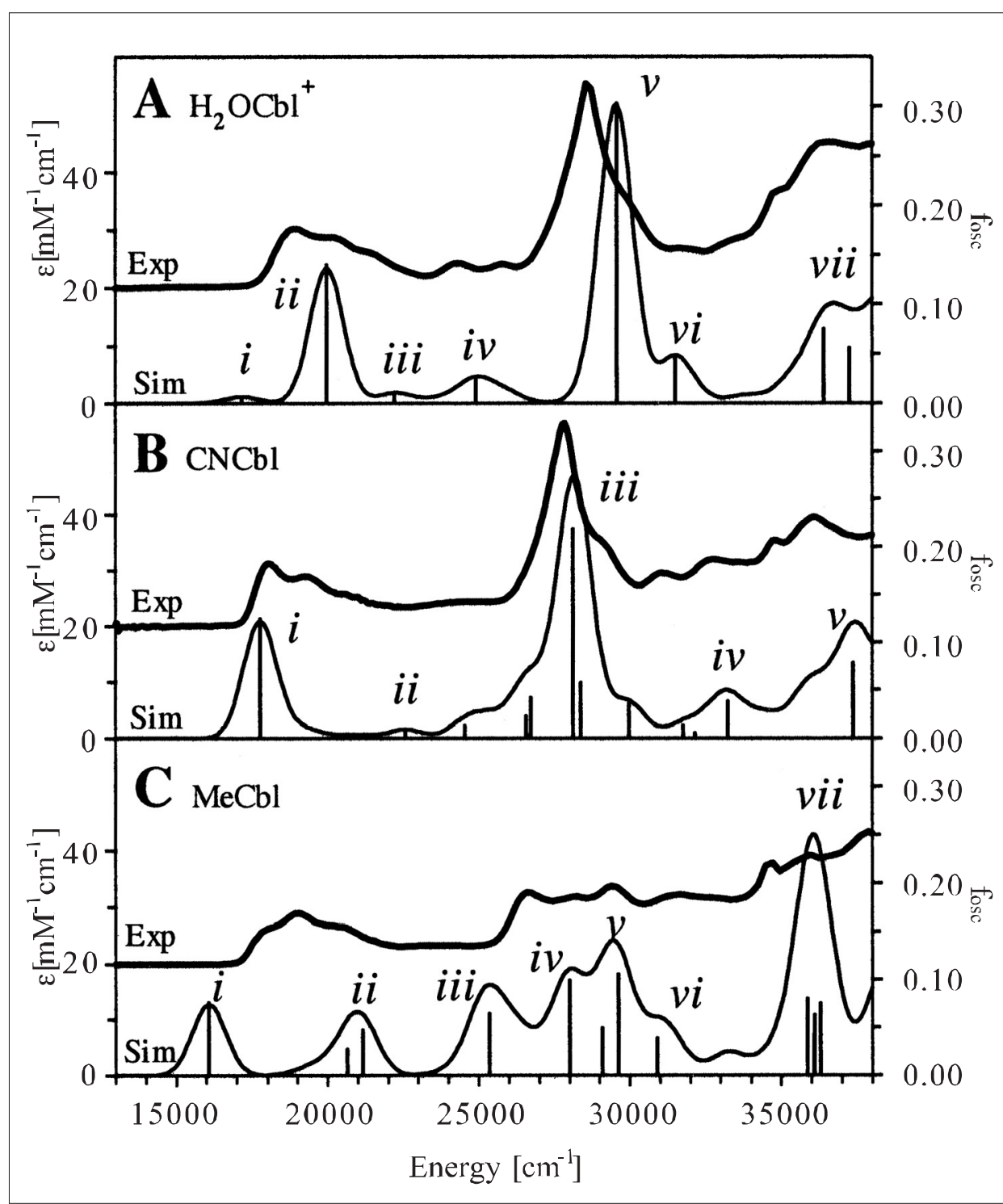

Fig. 6. Experimental and TD-DFT simulated Abs spectra for $\mathrm{H}_{2} \mathrm{OCbl}{ }^{+}(A), \mathrm{CNCbl}(B)$, and MeCbl (C). The calculated spectra were uniformly red-shifted by $5500 \mathrm{~cm}^{-1}$ to facilitate comparison with the experimental data. The transitions producing the dominant contributions to the calculated spectra are indicated by solid lines (the corresponding oscillator strengths are given on the right-hand axis). Reprinted with permission from [1]. Copyright 2003 American Chemical Society.

tra for $\mathrm{H}_{2} \mathrm{OCbl}^{+}$, $\mathrm{CNCbl}$, and $\mathrm{MeCbl}$ reproduce all essential features observed in the corresponding experimental data and properly predict the obvious experimental trends (such as the correlated shifts of the $\alpha / \beta$ and $\gamma$ bands and the intensity redistribution in the $\gamma$ region as a function of the upper axial ligand) permitted us to use the DFT calculated MO descriptions as the basis for assigning the relevant spectral features in both the 'typical' and the 'unique' Abs spectra of $\mathrm{Co}^{3+}$ Cbls. These assignments are briefly summarized below.

\section{Discussion}

Spectroscopic data of corrinoids reported in the literature are abundant. However, despite numerous decades of intensive in- vestigations of the $\mathrm{B}_{12}$ cofactors $\mathrm{MeCbl}$ and AdoCbl, their 'unique' Abs spectra remained largely unassigned [25]. Likewise, geometric and electronic factors distinguishing $\mathrm{Co}^{3+} \mathrm{Cbl}$ species exhibiting 'typical' Abs spectra from those displaying 'unique' Abs spectra had yet to be explored. To address these issues, we have developed a combined spectroscopic/computational methodology to probe the ground and excited electronic states of representative $\mathrm{Co}^{3+} \mathrm{Cbl}$ species exhibiting 'typical' and 'unique' Abs spectra [1]. Below, key findings from these studies are briefly discussed and their implications for biological $\mathrm{Co}-\mathrm{C}$ bond activation are explored.

$\mathrm{Co}^{3+} \mathrm{Cbl}$ Species Exhibiting 'Typical' Abs Spectra: Our TD-DFT-assisted analysis of the 'typical' $\mathrm{H}_{2} \mathrm{OCbl}^{+}$and $\mathrm{CNCbl} \mathrm{Abs}$ spectra (Fig. 3) reveals that the $\alpha$ and $\beta$ bands correspond to the electronic origin and the first member of a progression in $v_{L A}$ (depicted in the inset of Fig. 4) associated with the corrin-based HOMO $\rightarrow$ LUMO transition that is polarized along the $\mathrm{C}^{5} \cdots \mathrm{C}^{15}$ vector (Fig. 1). The most intense transition in the $\mathrm{D} / \mathrm{E}$ region is assigned to a corrin-based $\pi \rightarrow \pi^{*}$ transition from the HOMO-1 to the LUMO. Lastly, the single intense transition responsible for the prominent $\gamma$ band in these spectra is also attributed to a corrin-based $\pi \rightarrow \pi^{*}$ transition originating from the HOMO. The primary acceptor orbital in this transition is a corrin $\pi^{*}$ orbital that has the same symmetry as the HOMO with respect to the approximate $\mathrm{C}_{\mathrm{s}}$ mirror plane of the corrin macrocycle (Fig. 1), which results in a transition moment that is oriented roughly along the Co $\cdots \mathrm{C}^{10}$ vector.

As cyanide is a much stronger $\sigma$-donor than a water molecule, our DFT calculations predict that the Co $3 \mathrm{~d}_{2}$-based MO is considerably raised in energy in $\mathrm{CNCbl}$ relative to $\mathrm{H}_{2} \mathrm{OCbl}^{+}$, consistent with the $\sim 1.42$ $\mathrm{V}$ lower reduction midpoint potential of the former [44][45]. Additionally, the contribution from the formally unoccupied $\mathrm{Co} 3 \mathrm{~d}_{\mathrm{z}^{2}}$ orbital to the corrin-based HOMO increases from $0.2 \%$ in $\mathrm{H}_{2} \mathrm{OCbl}^{+}$to $1.5 \%$ in $\mathrm{CNCbl}$. This partial population of the $\mathrm{Co}$ $3 \mathrm{~d}_{\mathrm{z}^{2}}$ orbital induces a strong $\sigma$-antibonding interaction between the cobalt and the coordinated nitrogen of the lower axial base $\left(\mathrm{N}_{\mathrm{ax}}\right)$, thereby raising the energy of the HO$\mathrm{MO}$ and lowering the energies of electronic transitions originating from this orbital. Thus our DFT calculations afford an intuitively appealing model for explaining the long-established trend that the $\alpha / \beta$ and $\gamma$ bands in 'typical' $\mathrm{Co}^{3+} \mathrm{Cbl}$ Abs spectra uniformly shift to lower energy with increasing $\sigma$-donor strength of the upper axial ligand [25].

$\mathrm{Co}^{3+} \mathrm{Cbl}$ Species Exhibiting "Unique" Abs Spectra: Binding of an alkyl ligand in the upper axial position of $\mathrm{Co}^{3+} \mathrm{Cbls}$, as in $\mathrm{MeCbl}$ and AdoCbl, results in the appearance of a significantly perturbed, 'unique' $\mathrm{Co}^{3+} \mathrm{Cbl}$ Abs spectrum (Fig. 3). Our DFT computations on $\mathrm{MeCbl}$ indicate that these spectral changes reflect the increased $\sigma$ donor strength of the alkyl ligand and the consequent destabilization of all Co $3 \mathrm{~d}$ orbitals. As a result, the occupied Co $3 \mathrm{~d}$ orbitals of $\mathrm{MeCbl}$ and, presumably, alkylcobalamins in general, shift close in energy to the HOMO, which gives rise to (i) a net increase in the number of donor MOs available for electronic transitions and (ii) extensive mixing between Co $3 \mathrm{~d}$ and corrin $\pi$ orbitals. It is the combination of these two effects that is responsible for the 'unique' Abs spectra of alkylcobalamins.

The greater $\sigma$-donor strength of the upper axial ligand in $\mathrm{MeCbl}$ compared to $\mathrm{CNCbl}$ and $\mathrm{H}_{2} \mathrm{OCbl}^{+}$leads to a further in- 
crease in Co $3 \mathrm{~d}_{\mathrm{z} 2}$ orbital character in the corrin-based HOMO to $6.7 \%$, thereby inducing an even stronger $\mathrm{Co}-\mathrm{N}_{\mathrm{ax}} \sigma$-antibonding interaction. As a result, the DFT calculated $\mathrm{Co}-\mathrm{N}_{\mathrm{ax}}$ bond order decreases from 0.65 in $\mathrm{H}_{2} \mathrm{OCbl}^{+}$to 0.42 in $\mathrm{CNCbl}$ and 0.35 in $\mathrm{MeCbl}$. These values correlate nicely with the experimental $\mathrm{Co}-\mathrm{N}_{\mathrm{ax}}$ bond lengths that increase from $1.925 \AA$ in $\mathrm{H}_{2} \mathrm{OCbl}^{+}$[37] to $2.041 \AA$ in CNCbl [35] and $2.162 \AA$ in $\mathrm{MeCbl}$ [35]. Consequently, the model used to explain shifts in the $\alpha$ band position can also be invoked to rationalize the observed lengthening of the $\mathrm{Co}-\mathrm{N}_{\mathrm{ax}}$ bond with increasingly stronger $\sigma$-donors in the upper axial position [46].

Nature of the Co-C Bond and Implications for Co-C Bond Activation: The mechanism by which MeCbl- and AdoCbl-dependent enzymes activate the cofactor's $\mathrm{Co}-\mathrm{C}$ bond to produce a methyl cation and an $\mathrm{Ado}^{\circ}$ radical, respectively, is an enduring subject of intense research [20][28] [47-50]. Our DFT-generated electronicstructure description for $\mathrm{MeCbl}$ indicates that the $\mathrm{Co}-\mathrm{CH}_{3}$ bond is very covalent, as revealed by the large methyl $\mathrm{C} 2 \mathrm{p}_{\mathrm{z}}$ orbital character of $22 \%$ in the unoccupied Co $3 \mathrm{~d}_{\mathrm{z} 2}$-based MO. Since the methyl C $2 \mathrm{p}_{\mathrm{z}}$-derived occupied counterpart (i.e. the $\mathrm{Co}-\mathrm{C}$ $\sigma$-bonding MO) is in close energetic proximity to the corrin-based HOMO, these two MOs are strongly mixed. Consequently, the
HOMO of MeCbl exhibits substantial Co-C $\sigma$-bonding character, thus potentially offering the opportunity to modulate the properties of the organometallic bond through corrin ring deformations. However, because this electronic coupling only involves weak $\pi$-interactions, conformational changes of the corrin macrocycle are not expected to perturb the $\mathrm{Co}-\mathrm{CH}_{3}$ bond to any significant degree.

A puzzling and previously poorly understood result from earlier studies of $\mathrm{Co}^{3+}$ corrinoids is that while the identity of the upper axial ligand has a great effect on the $\mathrm{Co}-\mathrm{N}_{\mathrm{ax}}$ bond strength, the lower axial ligand does not appreciably modulate the $\mathrm{Co}-\mathrm{C}$ bond strength. Significantly, our DFT computations also provide a simple explanation for this phenomenon, summarized pictorially by the qualitative $\mathrm{MO}$ diagram in Fig. 7. While substitution of the upper axial ligand from $\mathrm{H}_{2} \mathrm{OCbl}^{+}$to $\mathrm{MeCbl}$ induces a substantial $\mathrm{Co}-\mathrm{N}_{\mathrm{ax}} \sigma$-antibonding interaction and, consequently, a weakening of this bond, replacement of the lower axial DMB ligand of $\mathrm{MeCbl}$ by a water molecule in methylcobinamide $\left(\mathrm{MeCbi}^{+}\right)$ has virtually no effect on the $\mathrm{Co}-\mathrm{C}$ bond (i.e. the bond order does not change, as both occupied MOs are $\sigma$-bonding with respect to the $\mathrm{Co}-\mathrm{C}$ bond). In support of this model, RR experiments by Spiro and coworkers revealed that the $\mathrm{Co}-\mathrm{C}$ bond strengths in

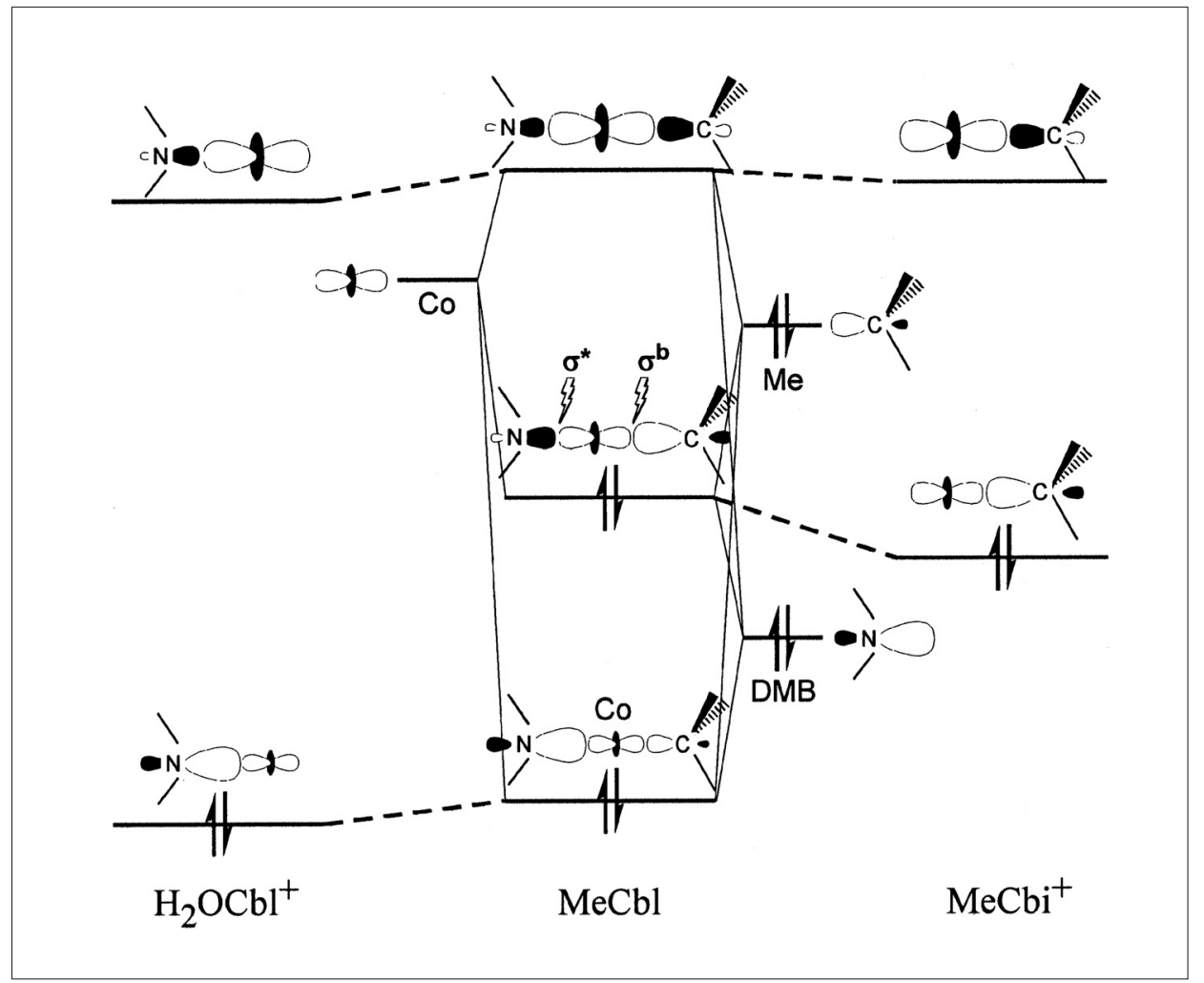

Fig. 7. Qualitative correlation diagram describing the bonding interactions between the cobalt center and the axial ligands in $\mathrm{H}_{2} \mathrm{OCbl}^{+}$(left), MeCbl (center), and $\mathrm{MeCbi}^{+}$(right). Reprinted with permission from [1]. Copyright 2003 American Chemical Society.
$\mathrm{MeCbl}$ and $\mathrm{MeCbi}^{+}$are virtually identical [30][31].

These results have important implications with respect to possible $\mathrm{Co}-\mathrm{C}$ bond activation mechanisms employed by $\mathrm{B}_{12^{-}}$ dependent enzymes, as they argue strongly against models invoking involvement of the lower axial ligand. For such a mechanism to be operative, it would be necessary for the $\sigma$-donor strength of the lower axial ligand to exceed that of the alkyl ligand, in which case the central MO of MeCbl in Fig. 7 would become $\sigma$-antibonding with respect to the $\mathrm{Co}-\mathrm{C}$ bond (rather than the $\mathrm{Co}-\mathrm{N}_{\mathrm{ax}}$ bond). However, this possibility can be ruled out since $\mathrm{X}$-ray structural data of $\mathrm{B}_{12^{-}}$ dependent enzymes reveal that the lower axial position is occupied by either the DMB or a histidine residue, both of which are considerably weaker $\sigma$-donors than alkyl ligands. Consequently, as more results become available, it seems less and less likely that enzymatic $\mathrm{Co}-\mathrm{C}$ bond activation involves corrin ring deformation and/or electronic perturbations induced by the lower axial ligand. Rather, a mechanism invoking stabilization of the $\mathrm{Co}-\mathrm{C}$ bond cleavage products; i.e. the $\mathrm{Co}^{1+} \mathrm{Cbl}$ species and the $\mathrm{Co}^{2+} \mathrm{Cbl} / \mathrm{Ado}^{\bullet}$ radical pair in the case of $\mathrm{MeCbl}$ and AdoCbl dependent enzymes, respectively, seems more plausible [51].

\section{Acknowledgment}

The author thanks Troy A. Stich and Amanda J. Brooks whose hard work this article reflects, and acknowledges the University of Wisconsin and the NSF (CAREER grant MCB0238530) for financial support of this project.

Received: February 13, 2004

[1] T.A. Stich, A.J. Brooks, N.R. Buan, T.C. Brunold, J. Am. Chem. Soc. 2003, 125, 5897-5914.

[2] R. Banerjee (Ed.), 'Chemistry and Biochemistry of B12', Wiley-Interscience, New York, 1999.

[3] R. Banerjee, Biochemistry 2001, 40, 6191-6198.

[4] D. Dolphin (Ed.), 'B12', Wiley, New York, 1982.

[5] M.L. Ludwig, C.L. Drennan, R.G. Matthews, Structure 1996, 4, 505-512.

[6] M.L. Ludwig, R.G. Matthews, Annu. Rev. Biochem. 1997, 66, 269-313.

[7] D.C.P.J. Hodgkin, J.H. Robertson, K.N Trueblood, R.J. Prosen, J.G. White, Nature (London) 1956, 176, 325.

[8] R.G. Finke, B.P. Hay, Inorg. Chem. 1984, 23, 3041

[9] R. Banerjee, Chem. Biol. 1997, 4, 175-186.

[10] R.G. Matthews, Acc. Chem. Res. 2001, 34, 681-689.

[11] C.L. Drennan, S. Huang, J.T. Drummond, R.G. Matthews, M.L. Ludwig, Science 1994, 266, 1669-1674.

[12] C.L. Drennan, R.G. Matthews, M.L. Ludwig, Curr. Opin. Struct. Biol. 1994, 4, 919-929. 
[13] N.H. Thoma, P.R. Evans, P.F. Leadlay, Biochemistry 2000, 39, 9213-9221.

[14] N.H. Thoma, P.F. Leadlay, Protein Sci. 1996, 5, 1922-1927.

[15] F. Mancia, P.R. Evans, Struct. Fold. Des. 1998, 6, 711-720.

[16] G.J. Gerfen, S. Licht, J.-P. Willems, B.M. Hoffman, J. Stubbe, J. Am. Chem. Soc. 1996, 118, 8192-8197.

[17] O.T. Magnusson, G.H. Reed, P.A. Frey, Biochemistry 2001, 40, 7773-7782.

[18] K.L. Brown, X. Zou, J. Inorg. Biochem. 1999, 77, 185-195.

[19] B.P. Hay, R.G. Finke, J. Am. Chem. Soc. 1986, 108, 4820-4829.

[20] S. Chowdhury, R. Banerjee, Biochemistry 2000, 39, 7998-8006.

[21] B.P. Hay, R.G. Finke, J. Am. Chem. Soc. 1987, 109, 8012-8018.

[22] Z. Schneider, A. Stroinski, 'Comprehensive B12: Chemistry, Biochemistry, Nutrition, Ecology and Medicine', De Gruyter, New York, 1987.

[23] R.A. Firth, H.A.O. Hill, J.M. Pratt, R.J.P. Williams, W.R. Jackson, Biochemistry 1967, 6, 2178-2189.

[24] A.M. Calafat, S. Taoka, J.M. Puckett, C. Semerad, H. Yan, L.B. Luo, H.L. Chen, R. Banerjee, L.G. Marzilli, Biochemistry 1995, 34, 14125-14130.

[25] J.M. Pratt, in 'Chemistry and Biochemistry of B12', Ed. R. Banerjee, Wiley, New York, 1999, pp 113-164.

[26] E.I. Solomon, M.L. Kirk, D.R. Gamelin, S. Pulver, Methods in Enzymology 1995, 246, 71-110.
[27] E.I. Solomon, M.A. Hanson, in 'Inorganic Electronic Structure and Spectroscopy', Eds. E.I. Solomon, A.B.P. Lever, Wiley, New York, 1999, Vol. 2, pp 1-130.

[28] F. Neese, E.I. Solomon, Inorg. Chem. 1999, 38, 1847-1865.

[29] S. Salama, T.G. Spiro, J. Raman Spec. 1977, 6, 57-60.

[30] S.L. Dong, R. Padmakumar, R. Banerjee, T.G. Spiro, J. Am. Chem. Soc. 1996, 118, 9182-9183.

[31] S.L. Dong, R. Padmakumar, R. Banerjee, T.G. Spiro, Inorg. Chim. Acta 1998, 270, 392-398.

[32] K.P. Jensen, S.P.A. Sauer, T. Liljefors, P.O Norrby, Organometallics 2001, 20, 550-556.

[33] M.P. Jensen, J. Halpern, J. Am. Chem. Soc. 1999, 121, 2181-2192.

[34] M.P. Jensen, D.M. Zinkl, J. Halpern, Inorg. Chem. 1999, 38, 2386-2393.

[35] L. Randaccio, M. Furlan, S. Geremia, M. Slouf, I. Srnova, D. Toffoli, Inorg. Chem. 2000, 39, 3403-3413.

[36] J.P. Bouquiere, J.L. Finney, M.S. Lehmann, P.F. Lindley, H.F.J. Savage, Acta Crystallogr. Sect. B-Struct. Commun. 1993, 49, 79-89.

[37] C. Kratky, G. Farber, K. Gruber, K. Wilson, Z. Dauter, H.F. Nolting, R. Konrat, B. Kräutler, J. Am. Chem. Soc. 1995, 117, 4654-4670.

[38] R. Bauernschmitt, R. Ahlrichs, Chem. Phys. Lett. 1996, 256, 454.

[39] E.M. Casida, C. Jamorski, K.C. Casida, D.R. Salahub, J. Chem. Phys. 1998, 108, 4439.
[40] R.E. Stratman, G.E. Scuseria, M.J. Frisch, J. Chem. Phys. 1998, 109, 8218.

[41] A.D. Becke, J. Chem. Phys. 1993, 98, 1372.

[42] A.D. Becke, J. Chem. Phys. 1993, 98, 5648.

[43] C. Lee, W. Yang, R.G. Parr, Phys. Rev. B 1988, 37, 785 .

[44] B. Jaselskis, H. Diehl, J. Am. Chem. Soc. 1954, 76, 4345-4348.

[45] O. Muller, G. Muller, Biochem. Zeit. 1962, 336, 299.

[46] J.M. Pratt, in 'Chemistry and Biochemistry of B12', Ed. R. Banerjee, Wiley, New York, 1999, pp 73-112.

[47] S. Chowdhury, R. Banerjee, J. Am. Chem. Soc. 2000, 122, 5417-5418.

[48] K.L. Brown, J. Zou, J. Am. Chem. Soc. 1998, 120, 9466-9474.

[49] S.L. Dong, R. Padmakumar, R. Banerjee, T.G. Spiro, J. Am. Chem. Soc. 1999, 121, 7063-7070.

[50] B. Kräutler, W. Keller, C. Kratky, J. Am. Chem. Soc. 1989, 111, 89368938.

[51] A.J. Brooks, M. Vlasie, R. Banerjee, T.C. Brunold, J. Am. Chem. Soc. 2004, submitted. 